\title{
MEKC Technique-Based Dairy Product Biochemical Analysis
}

\author{
Wei Wang* \\ Baotou Light Industry Vocational Technical College, Baotou, Inner Mongolia 014035, China
}

\begin{abstract}
The paper applies MEKC technique to simultaneously carry out quantitative analysis on dairy product's melamine (Mel) and 5-hydroxymethyl furfural (HMF) bi-component. The quantization analytic experiment starts with a systematic research on operating voltage, electrophoretic buffer solution gravity and optimum $\mathrm{pH}$ value, which further influences additives' concentration as well as other factors, and detects 10 kinds of practical samples on the basis of defined optimum conditions. Detection result indicates that both Mel and HMF showed peaks in 16min, simultaneously acquiring two indicator parameters in one analytical test, This test verifies applicability and scientific validity of MEKC technical approach in dairy product's Mel and HMF contents detection, which can play an effective role in providing rapid and reliable daily monitoring support for dairy industry.
\end{abstract}

Keywords: 5-hydroxymethyl furfural, capillary electrophoresis, dairy product, melamine, standard extract.

\section{INTRODUCTION}

Dairy product contains rich amino acids, which possess many benefits for consumers. If dairy products exceed the concentration of Mel, it can generate negative effects on consumers [1]. Generally, dairy product manufacturers apply ultra high temperature sterilization procession on products in order to extend shelf-life of the goods, but raw milk generates a series of unhealthy physicochemical and biological changes during the heating process, from which lactose and protein occur. HMF is an important intermediate product of Mailard's reaction, and if HMF exceeds the standard limit, it also generates negative effects on consumers [2]. Therefore, it is necessary to detect and monitor Mel content and HMF contents in dairy products. This paper thus presents one detection approach based on micelle electro kinetic capillary electrophoresis (MEKC) technique for the purpose of providing a convenient method for dairy product quantization detection analysis.

MEKC technique has been widely applicable in a variety of researches. Among which, Han Le et al. [3] (2013) applied MEKC-DAD approach simultaneously determining different batches of Scutellaria laterifolia and its processed products such as baicalin, scutellaria laterifolia element, wogonin, oroxylin A and apigenin's contents. It was pointed out that the approachwas simple and accuratewith good repeatability. Hu Jiang-Tao et al. [4] (2014) set up MEKC analytic approaches which simultaneously detected chilli powder, chilli oil and chilli sauce's Sudan Red I, II, III, IV, G. Yin Wan-Lu et al. (2014) [5] used MEKC analytic approach to detect vaccenic acid content in milk.

Zhang Li-Chun et al. [6] (2015) made use of a seasoning sorbic acid and benzoic acid contents MEKC measurement approach, tested and investigated detection wavelength, buffer solution selection, buffer solution $\mathrm{pH}$ values,

*Address correspondence to this author at the Baotou Light Industry Vocational Technical College, Baotou, Inner Mongolia 014035, China; Tel: +86-13674728328; E-mail: 13674728328@163.com separation voltage and column temperature as well as effects of other factors on separation. Luo Yi-Yuan et al. [7, 8] established an MEKC-DAD approach, simultaneously determining different origins and commodities' polygonum multiflorum's stilbene glucoside, emodin, aloe-emodin, rhein, physcion, and catechinic acid contents. Thereby, MEKC technique application feature is able to simultaneously detect the contents of various kinds of materials as compared to the current widely used gas chromatographymass spectrometry and liquid chromatography tandem mass spectrometry. Moreover, it is capable of overcoming high experiment expending, complicated operational process, long time-consuming as well as other drawbacks. Therefore, this research paper introduced MEKC technique for detecting Mel and HMF contents in dairy products in the hope of making an initial effort in the improvement and reformation of dairy product monitoring technique.

\section{EXPERIMENTAL DESIGN}

Capillary electrophoresis apparatus : Beckman P/ACE MDQ capillary electrophoresis system (Fullerton, CA) was used. Beckman company $32 \mathrm{Karat}^{\mathrm{TM}} 8.0$ version software workstation was applied. The equipment included a photodiode array detector which could scan at a scope of 190$600 \mathrm{~nm}$. Apparatus diagram is shown in Fig. (1) As can be seen, the structure consisted of a high voltage power supply, a capillary tube, a detector and two buffer solution storage bottles and sample cells that could support insertion at the two ends of the capillary tube and connection with power supply.

In electrolyte solution, charged particle transfer by electric field shifts from the opposite direction of its carried electrical charge at different speeds, is called electrophoresis. Capillary electrophoresis has a unified term of a kind of liquid phase separation technique that regards high voltage electric field as a driving force [8], uses capillary tube as a separation channel, and implements separation according to the samples' component mobility and allocation behavior 
differences. Capillary electrophoresis separation is a typical differential motion process. All factors that affect migration rate would also affect separation result, which provides an evidence for controlling and optimizing separation. Meanwhile, it also points out the need for developing a new mode for capillary electrophoresis separation. The new mode applied for separation in this research was micellar electrokinetic chromatography (MEKC).

Non-coating quartz capillary column of $75 \mu \mathrm{m}$ i.d., $375 \mu \mathrm{m}$ o.d., was provided by Polymicro Technologies, Phoenix, AZ, USA. Two kinds of electronic scales were selected and used, one was the Hunan instrument scale instrument limited company produced instrument with the scale measurement accuracy being $0.01 \mathrm{~g}$; while the other being Mettler Toledo instrument (Shanghai) limited company produced instrument with the scale measurement accuracy of $0.0001 \mathrm{~g}$.

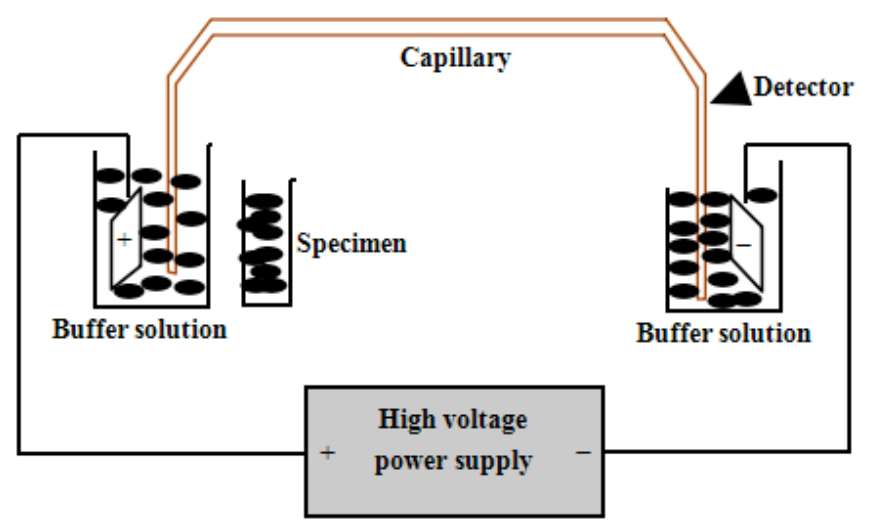

Fig. (1). Capillary electrophoresis apparatus configuration sketch.

Other instruments included ultrasonic cleaner, Centrifuge 5415D typed high speed centrifuge, XW-80A mini-sized vortex mixer, SG2typed $\mathrm{pH}$ meter and $0.45 \mu \mathrm{m}$ microfiltration membrane. Manufacturers of these instruments Tianjin
Automatic Science Instrument limited company, Eppendorf company, Shanghai Huxi Analysis Instrument limited company, Mettler-Toledo instrument (Shanghai) and Shanghai Bandao industrial limited company purification instrument factory.

Table 1 shows basic information of each experimental reagent. In Table 1, Mel manufacturer is Shanghai Sinopharm Chemical Reagent Limited Company, with its concentration above 99\%; HMF and Tris are purchased from Sigma-Aldrich (St.Louis, MO). Above all, reagents adopt ultrapure water preparation.

Standard stock solution preparation included dissolving $5 \mathrm{mg}$ Mel and HMF into $1 \%$ TCA solution so that $1 \mathrm{mg} / \mathrm{mL}$ Mel and HMF standard stock solution was achieved. The prepared $1 \mathrm{mg} / \mathrm{mL}$ standard stock solution was kept in the refrigerator at 4 degree centigrade, and it was made sure that these two solutions were be used in one month. TCA solution having $1 \%$ concentration was used to dilute $1 \mathrm{mg} / \mathrm{mL}$ Mel and HMF solutions, further diluting them into $0.05 \mu \mathrm{g} / \mathrm{ml}, \quad 0.10 \mu \mathrm{g} / \mathrm{ml}, \quad 0.20 \mu \mathrm{g} / \mathrm{ml}, \quad 0.50 \mu \mathrm{g} / \mathrm{ml}, \quad 2.0 \mu \mathrm{g} / \mathrm{ml}$, $5.0 \mu \mathrm{g} / \mathrm{ml}, 20.0 \mu \mathrm{g} / \mathrm{ml}$ and $100 \mu \mathrm{g} / \mathrm{ml}$ standard stock solutions.

In electrophoresis buffer solution preparation, the solution was composed of $15 \mathrm{mM}$ SDP, $15 \mathrm{mM}$ DSP and $80 \mathrm{mM}$ SDS. The $\mathrm{pH}$ value of the solution was adjusted to 6.85 , and then $0.45 \mu \mathrm{m}$ filter membrane was used for filtration, carrying out ultrasonic $10 \mathrm{~min}$ bubble removal treatment on solution after filtration. The solution was then maintained at room temperature, ensuring its availability in two weeks.

For liquid milk sample treatment, $1 \mathrm{~mL}$ liquid milk and $1 \mathrm{~mL} 4 \%$ TCA solution were blended into $50 \mathrm{~mL}$ centrifuge tube, and $2 \mathrm{~mL}$ ultrapure water was then added. Subsequently, vortex shaking $(2 \mathrm{~min})$ and ultrasound $(10 \mathrm{~min})$ were carried out. Interlayer clear solution was drawn and filtration was conducted in $0.45 \mu \mathrm{m}$ filter membrane. Filter liquor could directly implement the sample detection.

Table 1. Experiment reagent basic information list.

\begin{tabular}{|c|c|c|c|}
\hline ShiErWanJiLiuSuanNa & Sodium Dodecyl Sulfate & $\mathrm{C}_{12} \mathrm{H}_{25}-\mathrm{OSO}_{3} \mathrm{Na}$ & SDS \\
\hline BengSunNa & Sodium Tetraborate & $\mathrm{Na}_{2} \mathrm{~B}_{4} \mathrm{O}_{7} \cdot 10 \mathrm{H}_{2} \mathrm{O}$ & ST \\
\hline LinSuanErQingNa & Sodium Dihydrogen Phosphate & $\mathrm{NaH}_{2} \mathrm{PO}_{4}$ & SDP \\
\hline SanLuYiSuan & Trichloroa Cetic Acid & $\mathrm{Cl}_{3} \mathrm{CCOOH}$ & TCA \\
\hline QingYangHuaNa & Sodium Hydroxide & $\mathrm{NaOH}$ & $\mathrm{SH}$ \\
\hline YanSuan & Hydrochloric Acid & $\mathrm{HCl}$ & HA \\
\hline
\end{tabular}




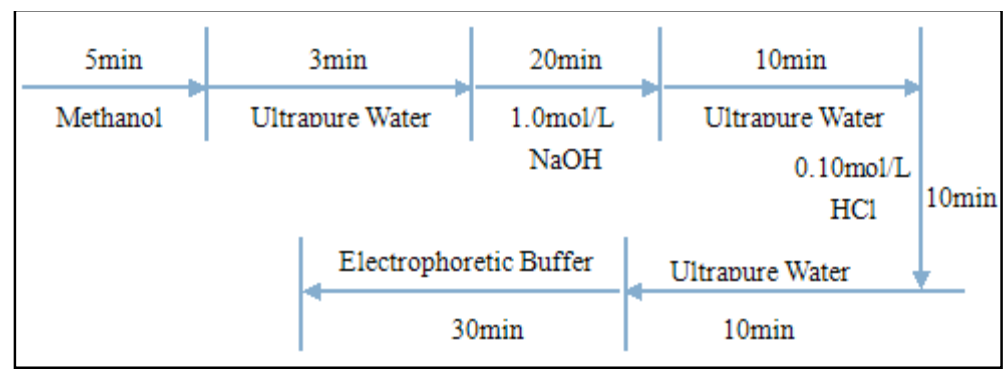

Fig. (2). New capillary tube handling and balancing flow.

Solid milk powder sample treatment involved dissolving of $2 \mathrm{~g}$ milk powder into $6 \mathrm{~mL} 1 \%$ TCA solution, successively carrying out vortex shaking for $2 \mathrm{~min}$ and ultrasound for $10 \mathrm{~min}$, from which $1 \%$ TCA solution had a constant volume of $10 \mathrm{~mL} .12000 \mathrm{rpm}$ centrifugal treatment was then undertaken for $4 \mathrm{~min}$, drawing an interlayer clear solution. $12000 \mathrm{rpm}$ centrifugal treatment was repeated on clear solution for $4 \mathrm{~min}$, and then again interlayer clear solution was drawn after centrifugation. Finally, filtration was performed on clear solution in a $0.45 \mu \mathrm{m}$ filter membrane. Filter liquor could directly implement the sample detection.

Before starting capillary electrophoresis, new capillary tube carried out handling and balancing as given in Fig. (2):

In case there are same applied samples to be detected, it should use electrophoresis buffer solution to wash them for 5 minutes ahead of time.

1) The experiment used capillary tube having a non-coated quartz, with an inner diameter of $75 \mu \mathrm{m}$, total length of $58.5 \mathrm{~cm}$, and valid length of $50 \mathrm{~cm}$.

2) $214 \mathrm{~nm}$ and $280 \mathrm{~nm}$ double -wavelengths were adopted to detect Mel and 5-HMF respectively.

3) Electrophoresis buffer solution $\mathrm{pH}$ value was 6.85 , which was compounded by $30 \mathrm{mmol} / \mathrm{L}$ PC and $80 \mathrm{mmol} / \mathrm{L}$ SDS.

The sample was introduced with an air pressure $(0.5 \mathrm{psi} \times 5 \mathrm{~s})$ and $15 \mathrm{kV}$ operating voltage. Capillary card case temperature was controlled at 25 degree centigrade.

4) Detected data formed drawing in $32 \mathrm{Karat}^{\mathrm{TM}} 8.0$ chromatographic work station after signal collection.

\section{RESULT ANALYSIS}

For optimization of Mel and HMF detection wavelengths, capillary electrophoresis apparatus diode array detector was adopted to carry out full wavelength scanning at a scope of 190-400nm, by obtaining Mel and HMF ultraviolet absorption spectrograms. By comparing, Mel and HMF maximum absorption wavelengths were defined as $202 \mathrm{~nm}$ and $280 \mathrm{~nm}$ respectively. The research, however, selected $214 \mathrm{~nm}$ and $280 \mathrm{~nm}$ respectively as Mel and HMF detection wavelengths. Fig. (3) illustrates that the two wavelengths detected electrophoresis images and two kinds of standard stock solution ultraviolet absorption spectrograms.

In Fig. (3), vertical axis represents absorbance, with the unit being mAU. As shown, standard stock solution was $5.0 \mu \mathrm{g} / \mathrm{mL}$ Mel standard substance and $5.0 \mu \mathrm{g} / \mathrm{mL} \mathrm{HMF}$ standard substance mixed solution. Electrophoresis buffer solution was $15 \mathrm{mM}$ SDP- $15 \mathrm{mM}$ DSP and $80 \mathrm{mM}$ SDS with $\mathrm{pH}$ value of 6.85 . Also, electrophoresis voltage wasis $15 \mathrm{kV}$ with $0.5 \mathrm{psix} 5 \mathrm{~s}$ sample introduction .

By Fig. (3), it is clear that capillary electrophoresis separation-DAD detection can well separate and distinguish Mel and HMF, apply its migration time and ultraviolet absorption characteristic spectrum to determine the nature of Mel and HMF, and also use peak area to carry out quantitative analysis.

1) Electrophoresis buffer solution type optimization: Buffer solution type experience directly affected working current, samples' separation degree, and peak shape and detection sensitivity and so on. By investigating THAM-PC buffer solution, ST buffer solution and SDP-DSP buffer solution of the three types, the paper respectively optimized the three different types of buffer systems' different concentrations and different $\mathrm{pH}$ values, finally adopting 30mM SDP-DSP buffer solution as an electrophoresis buffer system.

Electrophoresis buffer solution $\mathrm{pH}$ value optimization : Buffer solution $\mathrm{pH}$ value has a great impact on electro osmotic flow and target molecular carried charge, and exerts effects on Mel and HMF separation degree. By investigating SDP-DSP buffer solution with $\mathrm{pH}$ value of 6.70 to 7.00 , variation process impacts on target molecular carried charge and electro osmotic flow. The paper selected SDP-DSP buffer solution $\mathrm{pH}$ value as 6.85.

Additive concentration optimization: The paper defines SDS with $60 \mathrm{mmol} / \mathrm{L}-100 \mathrm{mmol} / \mathrm{L}$ concentration gradient to investigate additive influences during capillary electrophoresis separation process. During separation process, SDS composed micelle moves towards positive pole in capillary, and interacts with analyte through electrostatic function and formed hydrogen bond. Comprehensive analysis considered that SDS concentration impacts on separation degree, operating time, sensitivity, finally defining that $80 \mathrm{mmol} / \mathrm{L}$ is additive to SDS optimum concentration.

2) Separation voltage optimization: Operating voltage has important impacts on migration time of samples, working current and samples' each component separation degree. When column length is defined, operating voltage increases, and electro osmotic flow absolute value also increases, while migration time shortens. When voltage increases and goes beyond pole, joule heat influence intensifies, current gets excessively higher, and base line is not stable, which further reduces samples' separation de- 


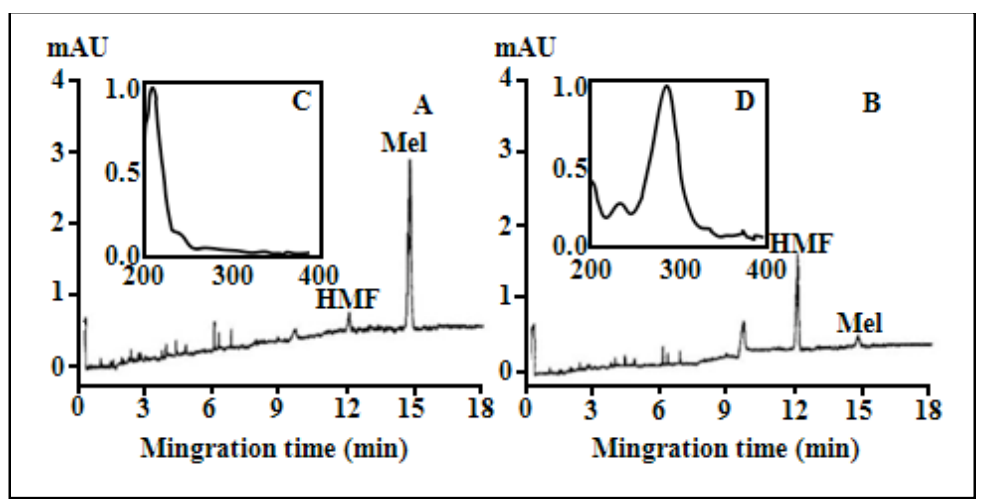

Fig. (3). Electrophoretic image (A: 214nm wavelength: B: 280nm wavelength) and ultraviolet absorption spectrum (C: Mel: D: HMF).

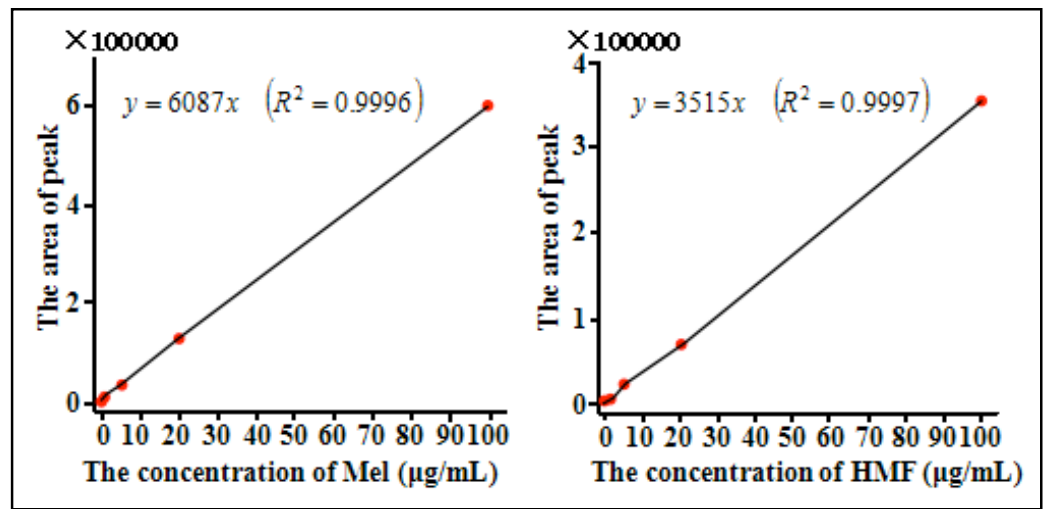

Fig. (4). Standard curve chart (Left: Mel: Right: HMF).

Table 2. Mel and HMF LOD, LOQ, LR and CC measurement result list.

\begin{tabular}{|c|c|c|c|c|}
\hline Compound & LOD $(\boldsymbol{\mu g} / \mathbf{m L})$ & LOQ $(\boldsymbol{\mu g} / \mathbf{m L})$ & LR $(\boldsymbol{\mu g} / \mathbf{m L})$ & $\mathbf{C C}\left(\boldsymbol{R}^{2}\right)$ \\
\hline \hline Mel & 0.047 & 0.05 & $0.05-100$ & 0.9996 \\
\hline HMF & 0.067 & 0.10 & $0.10-100$ & 0.9997 \\
\hline
\end{tabular}

gree. In order to separate Mel and HMF as well as other complicated ground substances in a short span of time, the research finally selected $15 \mathrm{kV}$ as the operating voltage.

To sum up, detection wave was applied at a $214 \mathrm{~nm}$ wavelength to detect $\mathrm{Mel}$, and at a $280 \mathrm{~nm}$ wavelength to detect HMF. Optimum $\mathrm{pH}$ value was defined as 6.85. Optimum SDP-DSP buffer solution concentration was $30 \mathrm{mmol} / \mathrm{L}$. Optimum SDS additive concentration was $80 \mathrm{mmol} / \mathrm{L}$. Optimum separation voltage was $15 \mathrm{kV}$.

$0.05 \mu \mathrm{g} / \mathrm{ml}, 0.10 \mu \mathrm{g} / \mathrm{ml}, 0.20 \mu \mathrm{g} / \mathrm{ml}, 0.50 \mu \mathrm{g} / \mathrm{ml}, 2.0 \mu \mathrm{g} /$ $\mathrm{ml}, 5.0 \mu \mathrm{g} / \mathrm{ml}, 20.0 \mu \mathrm{g} / \mathrm{ml}$ and $100 \mu \mathrm{g} / \mathrm{ml} \mathrm{Mel}$ and HMF standard solutions were selected to carry out capillary electrophoresis analysis, while taking $\mathrm{Mel}$ and HMF peak area as vertical coordinate, and regarding sample concentration as horizontal coordinate. Fig. (4) shows the standard curve chart.

By Fig. (4), it is clear that Mel had good linear relations at the concentration scope of $0.05-100 \mu \mathrm{g} / \mathrm{mL}$ and HMF had good linear relations at a concentration scope of $0.10-100$ $\mu \mathrm{g} / \mathrm{mL}$.
Table 2 shows Mel and HMF limits of detection LOD, limits of quantification LOQ, linear ranges LR and correlation coefficients CC. Among them, LOD value is 10 times blank sample introduction evaluation signal values plus 3 times' standard deviation; LOQ value is 10 times blank sample introduction evaluation signal values plus 10 times' standard deviation. The paper applies capillary electrophoresis -PDA which detects that Mel LOD is $0.047 \mu \mathrm{g} / \mathrm{mL}$, LOQ is $0.05 \mu \mathrm{g} / \mathrm{mL}$, while international reported milk Mel maximum residue limit is $1.0-2.5 \mathrm{mg} / \mathrm{kg}$. Therefore, the approach fully meets the demands of daily work's milk Mel detection. Besides, the approach also fully meets the demands of daily work's HMF detection.

Reproducibility research contains $\mathrm{Mel}$ and $\mathrm{HMF}$ forwarding time and peak area intraday relative standard deviation and interday relative standard deviation measurement. Among these, intraday deviation measures the same sample five times for sample pretreatment in the same day and undertakes capillary electrophoresis detection; inter-day deviation is carried out by continuously measuring the same sample for five days pretreatment and taking capillary electrophoresis detection. The purpose is to measure the accuracy 
Table 3. Mel and HMF's MT and PA reproducibility research result table.

\begin{tabular}{|c|c|c|c|}
\hline \multicolumn{2}{|c|}{ Sample } & MT (\%) & PA (\%) \\
\hline \hline \multirow{2}{*}{ Intraday $(\mathrm{n}=5)$} & Mel & 2.25 & 2.58 \\
\cline { 2 - 4 } & HMF & 1.69 & 3.53 \\
\hline \multirow{2}{*}{ Interday $(\mathrm{n}=5)$} & Mel & 4.30 & 4.61 \\
\cline { 2 - 4 } & HMF & 4.23 & 5.42 \\
\hline
\end{tabular}

Table 4. Milk Mel and HMF recovery research results table.

\begin{tabular}{|c|c|c|c|}
\hline \multirow{2}{*}{ Compound } & SC $(\mu \mathrm{g} / \mathbf{m L})$ & AR (\%) & RSD (\%) (n=4) \\
\hline \hline \multirow{2}{*}{ Mel } & 1.0 & 97.02 & 3.09 \\
\cline { 2 - 4 } & 5.0 & 94.85 & 3.20 \\
\cline { 2 - 4 } HMF & 1.0 & 99.20 & 3.11 \\
\cline { 2 - 4 } & 5.0 & 95.26 & 2.85 \\
\hline
\end{tabular}

of the evaluation approach. Table 3 shows the Mel and HMF migration time MF and peak area PA relative standard deviation. Table 3 data shows that the approach has good accuracy.

Approach recovery evaluation selected $\mathrm{Mel}$ and $\mathrm{HMF}$ with $1.0 \mu \mathrm{g} / \mathrm{mL}$ and $5.0 \mu \mathrm{g} / \mathrm{mL}$, two kinds ofspiked concentration (SC) to add a milk practical sample before sample treatment, for conducting a test according to the experimental design sample pretreatment approach and capillary electrophoresis detection approach, and for obtaining average recovery (AR) and relative standard deviation (RSD). Table 4 shows milk Mel and HMF recovery conditions.

By Table 4, it is clear that Mel average recovery is $95.95 \%$, HMF average recovery is $97.23 \%$, and the two components relative standard deviations are less than $5 \%$. Therefore, the approach recovery condition can be considered as good.

According to the experimental design, 10 kinds of dairy products are selected.Among them, seven kinds are liquid milk, and three are milk powder. The experiment optimization approach is adopted to carry out analytic detection on samples.

At first, TCA extracting solution is used to dilute, and then successively vortex shaking, ultrasonic extraction and centrifugal filtration are carried out, finally implementing capillary electrophoresis detection on samples.

As Fig. (5) shows typical milk practical samples extract liquor and extract liquor capillary electrophoretic image after respectively adding $5 \mu \mathrm{g} / \mathrm{mL}$ Mel and HMF. In practical samples, Mel and HMF concentrations are quantized through Mel and HMF peak areas. As far as liquid milk is concerned [9], concentration unit finally is conversed into $\mu \mathrm{g} / \mathrm{mL}$; as far as milk powder is concerned [10], concentration unit finally is conversed into $\mu \mathrm{g} / \mathrm{g}$. As Table 5 shows, capillary electrophoresis measured two kinds of different samples' Mel and HMF concentrations, from which comparison was carried out between Mel concentration and high performance liquid chromatography. High performance liquid chromatography approach measured data obtained through inspection and quarantine bureau handling with milk practical samples according to "People's Republic of China national standard GB/T22388-2008 raw milk and dairy product melamine detection approach" of Fujian province Quanzhou city [11] stipulating sample pretreatment procedures and then carrying out detection by high performance liquid chromatography. Carrying out comparison on (Table 5) CE-PDA and HPLCUV measuring ten kinds of samples' Mel concentrations, it can be found that CE-PDA measured concentration was slightly higher than that of HPLC-UV measurement. Data correlation analysis carried out on concentration, highlighted that CE-PDA measured concentration and HPLC-UV measured concentration data correlation coefficient was 0.99485 , which shows that two groups of data differences obtained by two approaches were mainly from system error. Two groups of data differences may be caused by two approaches' sample pretreatment differences. In national standard recommended raw milk and dairy product Mel detection approach, sample pretreatment also contains sample detection after carrying out solid phase extraction on extracting solution, as compared to capillary electrophoresis approach. However, extra solid phase extraction handling procedure may lead to samples' Mel recovery declination. The data provided by Fujian province Quanzhou city inspection and quarantine bureau shows that average recovery after solid phase extraction treatment is around $80 \%$.

By Table 5, following conclusions can be drawn:

1) Sample-1 is a kind of children drunk milk beverage, and use of capillary electrophoresis approach and high performance liquid chromatography approach did not detect Mel.

Addition of Sample-2 1010 $\mu \mathrm{g} / \mathrm{mL}$ Mel into Sample-1, capillary electrophoresis detection result was 10.26 $\mu \mathrm{g} / \mathrm{mL}$, which shows good accuracy and recovery of the approach to a certain extent. 
Table 5. Practical samples' Mel and HMF contents measurement result table.

\begin{tabular}{|c|c|c|c|c|}
\hline & Sample & \multicolumn{2}{|c|}{ Mel } & $\begin{array}{c}\text { HMF } \\
\begin{array}{r}\text { CE-DAD } \\
(\mu \mathrm{g} / \mathrm{mL})\end{array}\end{array}$ \\
\hline 1 & Milk drink for kids & l & l & 0.59 \\
\hline 2 & $\# 1$ spiked with $10 \mu \mathrm{g} / \mathrm{mL}$ Mel & 10.26 & 8.32 & 0.54 \\
\hline 4 & Ordinary milk, same brand as \#3 & 33.84 & 26.99 & 1.59 \\
\hline 5 & Baby milk powder & 20.10 & 16.95 & 2.25 \\
\hline 6 & Baby milk powder, same brand as $\# 5$, different batch & 23.63 & 18.18 & 1.27 \\
\hline 7 & Imported baby milk powder & / & / & 0.60 \\
\hline 10 & Soy milk powder & 31.73 & 26.80 & 0.91 \\
\hline
\end{tabular}

HPLC: The HPLC results of Mel were provided by Quanzhou Inspection and Quarantine Bureau of China, the mean of recoveries was $80 \%$. /: Not detected.

2) Sample-3 and Sample-4 are whole milk powder and milk beverage of the same brands', however Sample-4 contains higher concentration of Mel, as detected by capillary electrophoresis approach and high performance liquid chromatography.

Sample-5 and Sample- 6 respectively are milk powders of same brands belonging to different batches, and both were detected with Mel.

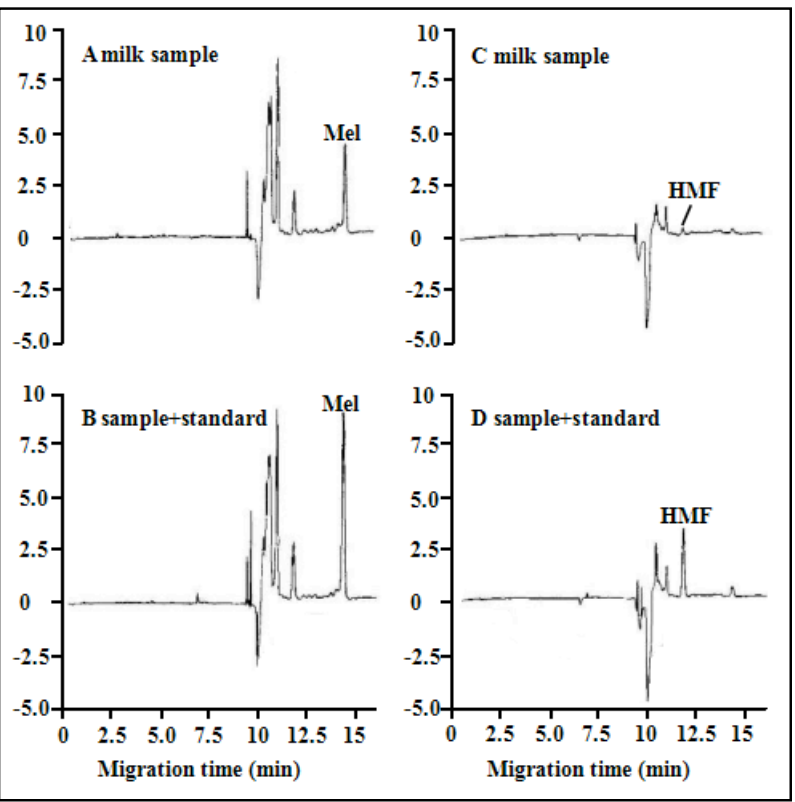

Fig. (5). Electrophoretic image of extracting solution that typical milk practical samples and same samples add standard substance (A and $\mathbf{B}$ is $214 \mathrm{~nm}$ wavelength detected spectrogram; C and $\mathbf{D}$ is $280 \mathrm{~nm}$ wavelength detected spectrogram).

3) Sample-7 and Sample-8 are imported infant milk powders. Mel is not detected in these two samples.
4) Sample-9 detects Mel with $1.32 \mu \mathrm{g} / \mathrm{g}$ concentration, which gets closer to national standard restrictive $\mathrm{Mel}$ maximum residue limit $(1.0-2.5 \mathrm{mg} / \mathrm{kg})$.

5) Sample-10 is a soybean milk powder, from which very high content of $\mathrm{Mel}$ is detected.

6) Sample-3 and Sample-9, two milk powders' HMF concentration was lower than $0.50 \mu \mathrm{g} / \mathrm{g}$, however the concentration cannot be accurately quantized. For other eight kinds of samples, HMF content concentration, was between $0.54-2.25 \mu \mathrm{g} / \mathrm{g}$, from which infant milk powder's HMF concentration was between $0.60-2.25 \mu \mathrm{g} / \mathrm{g}$, as a result conforming to document literature [10] liquid milk and milk powder HMF contents.

To sum up, MEKC detection technique is a kind of effective approach that can simultaneously analyze milk samples' Mel and HMF contents, and can provide reliable daily monitoring support for dairy industry.

\section{CONCLUSION}

The paper applies micellar electrokinetic capillary chromatography (MEKC) technique to detect dairy product melamine (Mel) and 5-hydroxymethyl furfural (HMF) contents. By detection results, it is clear that the technique approach has very strong applicability and scientific validity. Based on $\mathrm{MEKC}$ technique, dairy product biochemical analysis has the following three features:

1) Mel and HMF concentrations show peak values in $16 \mathrm{~min}$ and it meets food inspection rapidly gaining high efficiency demands.

2) The approach is simple and easy to operate, and can effectively save experiment cost.

3) It consumes little reagent equipment, and is a kind of economic and environmental friendly technical method. 


\section{CONFLICT OF INTEREST}

The author confirms that this article content has no conflict of interest.

\section{ACKNOWLEDGEMENTS}

Declared none.

\section{REFERENCES}

Z. Ping-Zhen, and S. Qi, "Food melamine attribute, harm and common inspection approach,"Scientific Practice, vol. 15, no. 3, pp. 282-283, 2014.

[2] L. Wen, Z. Xue-Mei, and L. Yang, " Low lactose milk browning suppression technique," Chinese Dairy Industry, vol. 22, no. 1, pp. 20-22, 2004.

[3] H. Le, C. Qiao-Xia, and L.Xun-Hong, "MEKC-DAD simultaneously scutellaria laterifolia six flavonoid constituent contents." $\mathrm{Me}$ dicine Analysis Magazine, vol. 33, no. 9, pp. 1525-1531, 2013.
[4] H. Jiang-Tao, Z.Wei, and C.Lu-Ying, "Micellar electrokinetic capillary chromatography analyzes chilli products' five kinds of Sudan Red,"Analysis Test Room, vol. 33, no. 12, pp. 1408-1412, 2014.

[5] Z. Li-Chun, G. Ke, and W. Lian, "Micellar electrokinetic chromatography approach rapid measure seasoning sorb and benzoic acid,“ Chinese Health Inspection Magazine, vol. 15, no. 10, pp. 1510-1512, 2015.

[6] L. Yi, L. Yi-Ping, and B. Yu, "Micellar electrokinetic chromatography simultaneously polygonum multiflorum's seven kinds of indicators constituents contents research, "Chinese Pharmaceutical Journal, vol. 50. no. 9, pp. 802-807, 2015 .

[7] L. Yi, L. Yi-Ping, and B. Yu, "Capillary tube zone electrophoresis high performance separation and high sensitivity detection $\alpha$ whey protein and $\beta$ - milk globulin," Research Bulletin of Analytical Chemistry, vol. 41, no. 10, pp. 1597-1600, 2013.

[8] L. Ly, M. Qing, and Z. Li-Jia, "Food common additives analysis and detection approach research development," Journal of Zunyi Normal College, vol. 16, no. 6, pp. 69-73, 2014,

(C) Wei Wang; Licensee Bentham Open.

This is an open access article licensed under the terms of the (https://creativecommons.org/licenses/by/4.0/legalcode), which permits unrestricted, noncommercial use, distribution and reproduction in any medium, provided the work is properly cited. 